\title{
Value of Tc99m (V) Dmsa Compared to Tc99m Mibi in Evaluation of Bone and Bone Marrow Lesions in Patients with Multiple Myeloma.
}

\author{
Shahenda S. Salem ${ }^{1}$ and Muhammed I. Ibrahim ${ }^{2}$. \\ ${ }^{1}$ Nuclear Medicine department, Cairo University, Cairo, Egypt., ${ }^{2}$ Clinical hematology department, \\ Mansoura University, Mansoura, Egypt.
}

Introduction: Multiple myeloma (MM) is primarily bone marrow neoplasm. Tc99m sestamibi (MIBI) is a potential imaging tracer in patients with multiple myeloma. Tc99m Pentavalent dimercaptosuccinic acid (V-DMSA) has an implication in imaging medullary carcinoma, head and neck tumors and soft tissue and bone tumors. Few reports evaluated imaging of multiple myeloma by Tc99m (V) DMSA and only one of them compared Tc99m (V) DMSA with Tc99m MIBI in assessment of chemotherapy effectiveness. Objective: Is to compare value of Tc99m (V) DMSA and Tc99m MIBI in detection of bone and bone marrow lesions in patients with multiple myeloma.

Materials and Methods: 32 patients with MM (18 M and $14 \mathrm{~F}$, mean age $55 \pm 9$ years) were enrolled in this study. Tc99m MIBI scan was done first followed 48 hours later by Tc99m (V) DMSA scan. Whole body Tc99m MIBI scan was obtained 10 minutes after IV injection of $555 \mathrm{MBq}$ of tracer. Whole body Tc99m (V) DMSA scan was acquired 2 hours following IV injection of $740 \mathrm{MBq}$ of tracer. Results: sensitivity, specificity, PPV, NPV and accuracy of Tc99m (V) DMSA was 93\%, 100\%, 100\%, 67\% and $94 \%$ versus 71\%, \%100, 100\%, $33 \%$ and $75 \%$ for Tc $99 \mathrm{~m} \mathrm{MIBI}$, respectively. Comparing pattern of bone and bone marrow involvement, it was focal in $18(56 \%)$ patients, diffuse in $5(16 \%)$ patients, diffuse and focal in $3(9 \%)$ patients and normal in $6(19 \%)$ patients in Tc99m (V) DMSA scans, while, it was focal in $6(19 \%)$ patients, diffuse in $11(34 \%)$ patients, focal and diffuse in $3(9 \%)$ patients and normal in $12(38 \%)$ patients in Tc99m MIBI scans.

Conclusion: Tc99m (V) DMSA is better than Tc99m MIBI in evaluation of bone and bone marrow in patients with MM. Tc99m (V) DMSA performed better in detection of focal lesions, whereas Tc99m MIBI was superior in visualization of diffuse disease.

Key words: Multiple myeloma, Tc99m sestamibi, Tc99m Pentavalent dimercaptosuccinic acid, bone and bone marrow involvement.

Corresponding Author: Dr. Shahenda S. Salem

Email: afssalem@yahoo.com

\section{INTRODUCTION}

Multiple Myeloma (MM) is a primarily bone marrow neoplasm that is characterized by proliferation of monoclonal myeloma cells with possible bone and soft tissue extension ${ }^{1}$.

In most institutions; conventional radiograph is still considered to be the golden standard to evaluate disease stage, therapy response and follow up. However, a false negative rate of $30-70 \%$ is considered a serious limitation of skeletal survey, which leads to significant underestimation in diagnosing and staging of patients with multiple myeloma².

Bone scintigraphy has some limitations in detecting bone lesions at the time of diagnosis since Tc99m MDP scintigraphy does not trace lesions lacking osteoblastic activity $^{3}$.
Tc99m Sestamibi (MIBI) has been proposed as a potential tracer in patients with multiple myeloma, as its increased uptake in the bone marrow has been reported as an indicator of myeloma activity ${ }^{4}$ It has been suggested to be used in staging and follow up of patients with multiple myeloma with a high sensitivity and specificity ${ }^{5}$.

Tc99m Pentavalent dimercaptosuccinic acid (V-DMSA) has been shown to have a clinical usefulness in imaging some malignant tumors such as medullary thyroid carcinoma, head and neck tumors as well as soft tissue and bone tumors ${ }^{6}$.

Imaging of multiple myeloma by Tc99m (V) DMSA has also been reported by Athanasoulis et $\mathrm{al}^{7}$ Athanasoulis et al. ${ }^{8}$ also stated that Tc99m (V) DMSA 
scan may become a useful diagnostic tool in the detection of osteolytic Tc99m MDP negative bone metastases $^{8}$. However, a little data have been published to compare between Tc99m (V) DMSA and Tc99m MIBI in detection of bone and bone marrow lesions in multiple myeloma.

Objective: Is to evaluate compare Tc99m (V) DMSA with Tc99m MIBI in the detection of bone and bone marrow lesions in patients with multiple myeloma.

\section{MATERIALS AND METHODS}

Thirty two consecutive patients with multiple myeloma (18 males and 14 females, mean age $55 \pm 9$ years) were referred to nuclear medicine department (NEMROK), Cairo University and were prospectively enrolled in the period from January, 2010 till June, 2010 in this study. Patient inclusion and research protocol was approved by the ethical committee. All patients had an informed consent before examinations.

Initial diagnosis and staging were made according to standard criteria ${ }^{9}$. The patients were clinically and biochemically evaluated at the time of the scan to determine their clinical status and disease activity. The patients were followed clinically after the scan for at least 12 months with complete knowledge of their laboratory and radiological follow up results.

\section{Imaging Acquisition:}

Tc99m MIBI Imaging: Tc99m MIBI scan was acquired first in all patients followed 48 hours later by Tc99m (V) DMSA scan to avoid carryover of image data. Images were acquired on a dual head gamma camera equipped with low energy general purpose collimator (ADAC/PHILIPS Vertex plus) using a $20 \%$ window centered at $140 \mathrm{Kev}$ and coupled with a dedicated computer system. Anterior and posterior whole body scans were acquired for all patients to improve the sensitivity of detecting small lesions.

Tc99m MIBI was prepared according to the manufacturer's instructions. Each patient received 555 $\mathrm{MBq}$ of Tc99m MIBI intravenously and anterior and posterior whole body scan was obtained 10 minutes after the injection ${ }^{10}$. The early scans were acquired at 10 minutes to avoid vertebral and pelvic shielding caused by biliary excretion and over time decrease of tracer bone uptake, which reported to occur in about half of the patients, probably influenced by the mechanism of active extrusion as well as over expression of P-glycoprotein associated with multidrug resistance.

\section{Tc99m (V) DMSA Imaging:}

Tc99m (V) DMSA was prepared by adding 7\% sodium bicarbonate to the commercial DMSA kit. Anterior and posterior whole body scans were acquired 2 hours following intravenous injection of $740 \mathrm{MBq}$ of the tracer. All scintigrams were obtained on the same dual head gamma camera (ADAC/PHILIPS Vertex plus) connected to low energy high resolution collimator using a $20 \%$ window centered at $140 \mathrm{Kev}$ and coupled with a dedicated computer system ${ }^{11}$.

\section{Imaging Interpretation:}

Both sets of Tc99m (V) DMSA and Tc99m MIBI scans were calcified into four classes:

1. Normal (only physiologic uptake i.e. the nasopharynx, axial skeleton, breast, liver, spleen, heart, kidneys, urinary bladder, great vessels and skeletal muscles for Tc-99m V DMSA and in the heart, liver and spleen for Tc99m MIBI).

2. Diffuse (diffuse bone marrow uptake in the axial and appendicular skeleton).

3. Focal uptake (areas of focal uptake of the radiotracer were seen).

4. Focal plus diffuse uptake (when both patterns were evident $)^{12}$.

The results of both sets of Tc99m (V) DMSA and Tc99m MIBI scans for each patient were correlated with a recent skeletal survey as a gold standard and the biochemical and clinical data were also assessed to detect disease activity.

\section{Statistical Analysis:}

Data were statistically analyzed using the SPSS 16 Software (SSPS incorporation, Chicago, USA). Quantitative data were summarized and expressed as mean+standard deviation, whereas qualitative data were expressed as frequency. Sensitivity, specificity, positive predictive value (PPV), Negative Predictive Value (NPV) and accuracy of both studies were calculated. Groups included in the study were compared using the Kruskall-Wallis test and each of the two groups was then compared using the MannWhitney test. Significance was set at the value of less than 0.05 level.

\section{RESULTS}

According to clinical staging of the patients; two were at stage II and 30 were stage III ( 24 were IIIA 
and 6 were IIIB). The mean time since the disease onset was $29.12+16.3$ months. Fourteen patients were previously treated with radiochemotherapy and phosphate injections. Three patients were treated with radiochemotherapy, 9 patients were treated with chemotherapy and phosphate injection and 6 patients were treated with only chemotherapy. The patients were treated between 1 and 2 years before the study. The patients were referred to our center because of uncomplicated bony pains in fifteen patients (47\%), bony pains with associated fracture in 2 patients $(6 \%)$, bony pains with cord compression in 3 patients $(9 \%)$ and bony pain and anemia in 3 patients (9\%). Anemia was the sole presentation in 4 patients $(13 \%)$, while hypercalcemia was the presentation in 5 patients $(16 \%)$.

Of the 32 patients performed Tc99m (V) DMSA scans were positive in 26 patients and negative in 6 patients; 2 patients were false negative and 4 patients were true negative. While, Tc99m MIBI, 20 patients had positive scans and 12 patients had negative scans; 8 patients were false negative and 4 patients were true negative. No false positive results were found. No false positive results were also found (Table 1). The sensitivity, specificity, PPV, NPV and accuracy of Tc99m (V) DMSA was 93\%, 100\%, 100\%, 67\% and $94 \%$ Versus $71 \%, \% 100,100 \%, 33 \%$ and $75 \%$ for Tc99m MIBI, respectively (Table 2).

Comparing the pattern of bone and bone marrow uptake in both scans, it was focal in $18(56 \%)$ patients, diffuse in $5(16 \%)$ patients, diffuse+focal in $3(9 \%)$ patients and normal in $6(19 \%)$ patients in Tc99m (V) DMSA scans, while, it was focal in $6(19 \%)$ patients, diffuse in $11(34 \%)$ patients, diffuse+focal in $3(9 \%)$ patients and normal in $12(38 \%)$ patients in Tc99m

Table 1: Results of Tc99m MIBI scan in comparison to skeletal X-ray survey in detecting bone lesions.

\begin{tabular}{lcccc}
\hline & TP & TN & FP & FN \\
\cline { 2 - 5 } Tc99m (V) DMSA & 26 & 4 & 0 & 2 \\
\hline Tc99m MIBI & 20 & 4 & & 8
\end{tabular}

TP: true positive, TN: true negative, FP: false positive, FN: false negative.
MIBI scans (Table 3, Figure 1). Regarding the pattern of bone and bone marrow involvement in Tc99m (V) DMSA and Tc99m MIBI scans, a significant statistical difference between the 2 imaging methods was found, in particularly, Tc99m (V) DMSA detected focal pattern with higher frequency than Tc99m MIBI $(P<0.001)$. On the contrary, Tc99m MIBI detected diffuse pattern with a higher frequency than Tc99m (V) DMSA $(P<0.001)$.

Six patients with focal bone marrow involvement in Tc99m (V) DMSA showed diffuse activity in Tc99m MIBI scan. Both Tc99m (V) DMSA and the Tc99m MIBI scan revealed the same myelomatous infiltration pattern; normal in 6 patients, focal in 6 patients, diffuse in 5 patients and diffuse+focal in 3 patients. Finally 6 patients with focal pattern on Tc99m (V) DMSA were normal on Tc99m MIBI (Figure 2).

Correlating the results of both Tc99m (V) DMSA and Tc99m MIBI scans with disease activity of the patients; Tc99m MIBI showed positive uptake in 20 patients and were all positive in Tc99m (V) DMSA scan with evidence of active disease. Four of the 12 patients with a negative result on Tc99m MIBI scans were in remission and were also negative in Tc99m (V) DMSA scan. However, the other 8 patients had an active disease and 6 of them showed positive myelomatous bone marrow deposits on Tc99m (V) DMSA scan. While, the remaining two patients were negative Tc99m (V) DMSA scan in spite of disease activity.

Finally, Tc99m (V) DMSA upstaged and modified the clinical management in 6 patients $(19 \%)$, found to be positive on Tc99m (V) DMSA and negative on Tc99m MIBI scans.

Table 2: Sensitivity, specificity, positive predictive value, negative predictive value and accuracy of Tc $99 \mathrm{~m}(\mathrm{~V})$ DMSA scans compared to Tc $99 \mathrm{~m}$ MIBI scans in evaluation of bone lesions in patients with multiple myeloma.

\begin{tabular}{lcc}
\hline & Tc99m (V) DMSA & Tc99m MIBI \\
\cline { 2 - 3 } sensitivity & $93 \%$ & $71 \%$ \\
\hline specificity & $100 \%$ & $100 \%$ \\
\hline PPV & $100 \%$ & $100 \%$ \\
\hline NPV & $67 \%$ & $33 \%$ \\
\hline Accuracy & $94 \%$ & $75 \%$ \\
\hline PPV: positive predictive value, NPV: negative predictive value.
\end{tabular}


Table 3: Pattern of bone and bone marrow findings in both Tc99m (V) DMSA and Tc99m MIBI scans.

\begin{tabular}{lcccc}
\hline & N & F & D & F+D \\
\cline { 2 - 5 } Te99m (V) DMSA & $6(19 \%)$ & $18(56 \%)$ & $5(16 \%)$ & $3(9 \%)$ \\
\hline Tc99m MIBI & $12(38 \%)$ & $6(19 \%)$ & $11(34 \%)$ & $3(9 \%)$
\end{tabular}

N: normal, F: focal, D: diffuse, F+D: focal+diffuse.

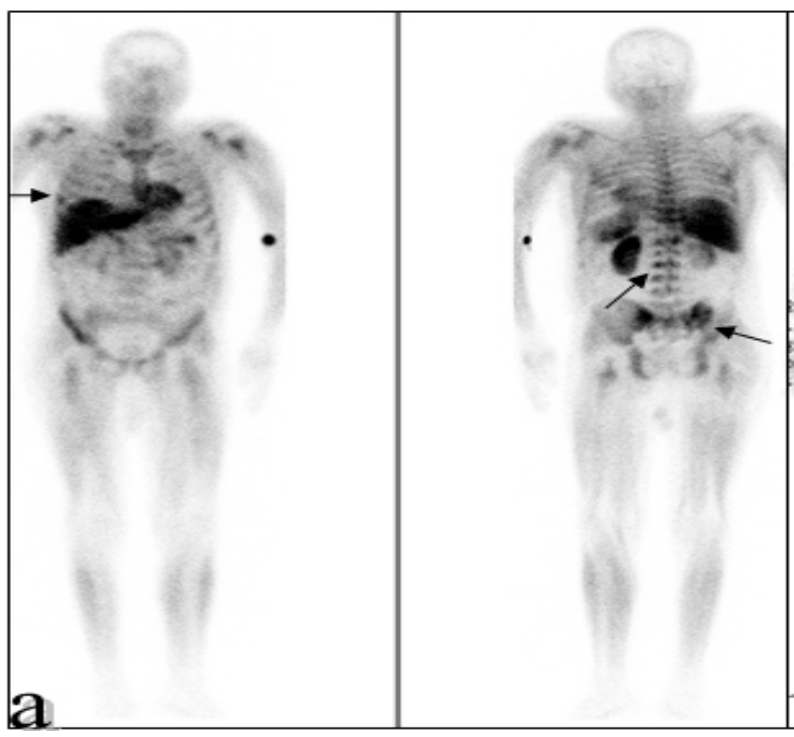

Figure 1 (A): 42 years old patient with previously treated multiple myeloma showing diffuse + focal pattern of uptake in both Tc99m MIBI and Tc99m (V) DMSA scans (a) Anterior and posterior whole body Tc99m MIBI scan shows diffuse marrow uptake with focal rib, vertebral and pelvic lesions [arrows].

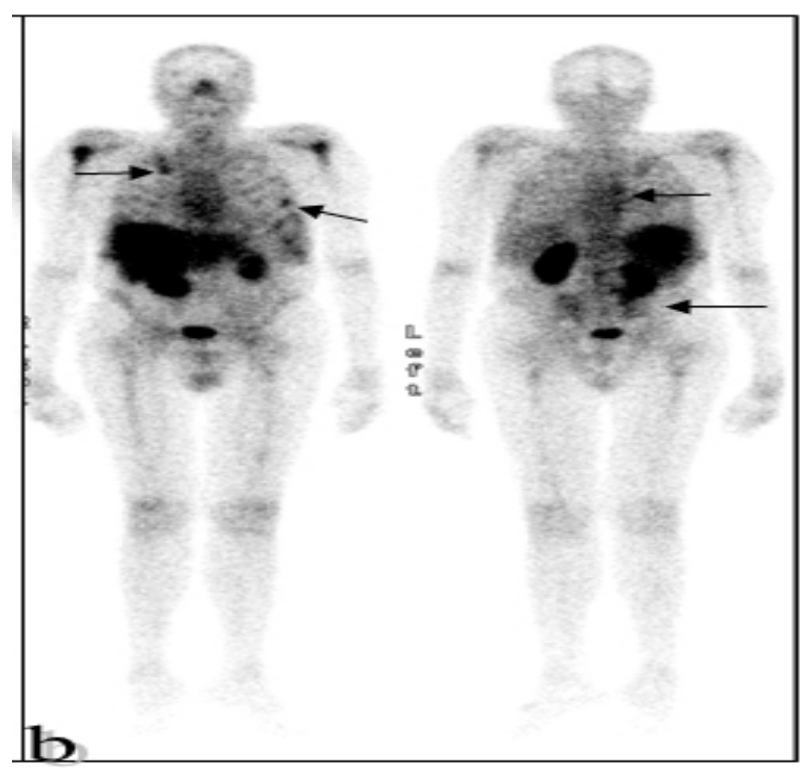

Figure 1 (B): Anterior and posterior whole body Tc99m (V) DMSA scans show diffuse marrow uptake with focal rib, vertebral and pelvic lesions [arrows].

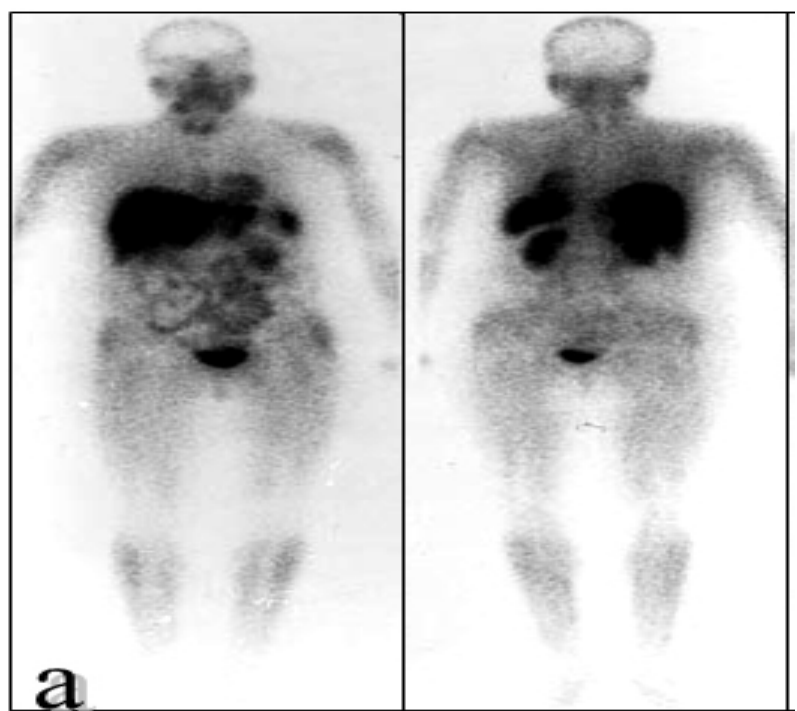

Figure 2 (A): 53 years old patient with previously treated multiple myeloma showing focal pattern on Tc99m (V) DMSA and normal on Tc99m MIBI scan (a): Anterior and posterior whole body Tc99m MIBI scan shows normal pattern of uptake

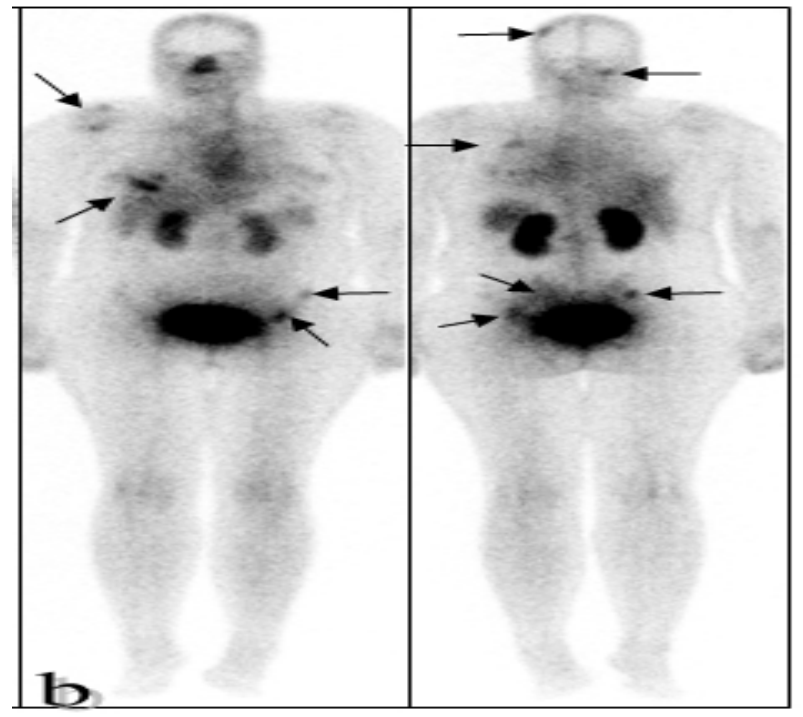

Figure. 2 (B): Anterior and posterior whole body Tc99m (V) DMSA scans show multiple focal bone marrow lesions in multiple skull bones, ribs, right shoulder and multiple pelvic bones [arrows].

\section{DISCUSSION}

Conventional X-ray is the standard imaging technique in evaluation of patients with multiple myeloma. However, particularly in previously treated patients, X-ray has a serious limitation to identify bone lesions corresponding to sites no longer active because of successful therapy. Tc99m MDP is less sensitive than X-ray in detecting skeletal lesions in patients with $\mathrm{MM}$ as it is not capable of evaluating bone marrow disease activity owing to lack of osteoblastic response usually produced in bone ${ }^{3}$. Tc99m 
MIBI appeared to overcome this limitation, reliably detecting active bone lesions in MM patients. It also has been reported to be more specific than conventional $\mathrm{X}$-ray in detection of active focal lesions in previously treated MM patients. In a prospective study by Alper et al. to determine the potential of Tc99m MIBI imaging for evaluation of extent of primary disease in 20 patients with advanced stage MM, compared with skeletal survey and bone scintigraphy. All Tc99m MIBI scans were positive for the presence of active MM, whereas skeletal surveys were positive in 18 patients $(90 \%)$ with osteolytic lesions. Bone scintigraphy demonstrated MM in only 15 patients $(75 \%)^{10}$.

Both Tc99m MIBI and Tc99m (V) DMSA have been reported as tumor imaging agents for many neoplasm and bone metastases including multiple myeloma ${ }^{11-13}$.

Tc99m MIBI accumulated in the viable tumor cells due to high cellularity and mitochondrial activation. Dimopoulos et al. reported localization of Tc99m MIBI inside the plasma cells infiltrating the bone marrow. Additionally, bone marrow biopsy showed linear correlation with bone marrow uptake of Tc99m MIBI ${ }^{14}$.

Tc99m (V) DMSA has a different mechanism of tumor accumulation than that of Tc99m MIBI. The high glycolytic rate of the malignant tissues results in increase in the production of lactic acid, hence the acidic $\mathrm{pH}$ of the tumor. Acidification of the tissues causes accumulation of Tc99m (V) DMSA ${ }^{15}$. Denoyer et al. recently reported that Tc99m (V) DMSA uptake in tumor cell lines is mediated by $\mathrm{NaPi}$ cotransporter type $\mathrm{III}^{16}$. Others demonstrated that Tc99m(V)DMSA is a marker of cell viability like $18 \mathrm{~F}$-fluorodeoxyglucose (FDG) and Tc99m MIBI, as detection of tumor viability is important in assessment of treatment efficacy ${ }^{17}$ as well as follow-up of cancer patients ${ }^{4}$.

The metabolic pathway of Tc99m (V) DMSA, which reflects the type III NaPi co-transporter activity is different from that of FDG, which follows the glucose transport and the glycolytic pathway. Therefore, Tc99m (V) DMSA could provide additional information on the metabolic status of tumors after treatment.

Some studies have suggested a potential role for MIBI scintigraphy during monitoring of cancer patients receiving and after chemotherapy ${ }^{18}$. However, Tc99m MIBI is also a probe of multidrug resistance (MDR) phenotype, which may interfere with determination of tumor activity ${ }^{19,20}$. In their work, Denoyer, et al. demonstrated that Tc99m (V) DMSA was independent of chemoresistance mediated by the major efflux pumps expressed in cancer cells and by the high levels of P-glycoprotein and MRP1. The authors assumed that Tc99m (V) DMSA could provide further information on viability of tumors with MDR phenotype and Tc99m (V) DMSA could therefore be considered as a useful non-invasive tool for follow-up of resistant tumors ${ }^{21}$.

In the current study, we compared between Tc99m (V) DMSA and Tc99m MIBI in the evaluation of bone involvement in patients with multiple myeloma. Our findings showed that the sensitivity, specificity, PPV, NPV and accuracy for Tc99m (V) DMSA was 93\%, $100 \%, 100 \%, 67 \%$ \& $94 \%$ compared to $71 \%, \% 100$, $100 \%, 33 \%$ and $75 \%$ for Tc $99 \mathrm{~m}$ MIBI, respectively. These results showed a better sensitivity, NPV and accuracy for Tc99m (V) DMSA compared to Tc99m MIBI scan with the same specificity and PPV. Our results go hand in hand with a recent study published by Mele et al., who reported a sensitivity of $77 \%$ for MIBI scan in a multicentre study evaluated 397 whole body scans done for patients with $\mathrm{MM}^{22}$. However, earlier studies reported higher sensitivity of MIBI ranged from 90 to $100 \%$, with the same specificity ( 88 to $96 \%)^{23,24}$. The intense hepatosplenic tracer uptake is possibly obscuring active and also small lesions in these localized areas. Finally, in Tc99m MIBI studies overexpression of P-glycoprotein associated with multidrug resistance could lower the lesion detection rate; hence its sensitivity as it has been shown that Tc99m MIBI is actively eliminated from the cell by P-glycoprotein.

Comparing the pattern of bone and bone marrow involvement on Tc99m (V) DMSA and Tc99m MIBI scans; Tc99m (V) DMSA detected focal lesions in higher frequency than Tc-99m MIBI with a significant statistical difference between the 2 imaging methods $(P<0.001)$. On the contrary, Tc99m MIBI detected diffuse pattern in higher frequency than Tc99m (V) DMSA $(P<0.005)$.

In a study done by Fonti et al., Tc99m MIBI and MRI were found to be superior to FDG PET/CT for visualization of diffuse disease. Meanwhile, FDG PET/ CT performed better than Tc99m MIBI and MRI in detection of focal lesions ${ }^{25}$.

Finally, Tc99m (V) DMSA upstaged and modified the clinical management in 6 patients $(19 \%)$ by confirming osseous and bone marrow involvement in context of active disease.

\section{CONCLUSION}

Tc99m (V) DMSA is better than Tc99m MIBI in evaluation of bone and bone marrow lesions in patients with multiple myeloma and its uptake correlated with disease activity. Unlike Tc99m MIBI, Tc99m (V) DMSA is not affected by the MDR phenomenon; however, correlation with immunohistochemical analysis of $\mathrm{P}$ glycoprotein in tumor cells is recommended. Tc99m 
(V) DMSA performed better than Tc99m MIBI in the detection of focal lesions, whereas Tc99m MIBI was superior in the visualization of diffuse disease.

\section{REFERENCE}

1. Nanni C, Zamagni E, Farsad M, Castellucci P, Tosi $\mathrm{P}$, Cangini $\mathrm{D}$, et al. Role of 18F-FDG PET/CT in the assessment of bone involvement in newly diagnosed multiple myeloma: Preliminary results. Eur.J.Nucl.Med. Mol.Imaging 2006;33(5):525-31.

2. Baur Melnyk A, Buhmann S, Becker C, Schoenberg $\mathrm{SO}$, Lang $\mathrm{N}$, Bartl $\mathrm{R}$, et al. Whole-body MRI versus whole-body MDCT for staging of multiple myeloma. Am.J.Roentgenol. 2008;190(4):1097-104.

3. Catalano L, Pace L, Califano C, Pinto AM, De Renzo A, Di Gennaro F, et al. Detection of focal myeloma lesions by technetium-99m-sestaMIBI scintigraphy. Haematologica 1999;84(2):119-24.

4. Pace L, Catalano L, Del Vecchio S, Di Gennaro F, De Renzo A, Sica G, et al. Predictive value of technetium-99m sestamibi in patients with multiple myeloma and potential role in the follow-up. Eur.J.Nucl.Med. 2001;28(3):304-12.

5. Villa G, Balleari E, Carletto M, Grosso M, Clavio M, Piccardo A, et al. Staging and therapy monitoring of multiple myeloma by $99 \mathrm{mTc}$-sestamibi scintigraphy: A five year single center experience. J.Exp.Clin.Cancer Res. 2005;24(3):355-61.

6. Zissimopoulos A, Zanglis A, Andreopoulos D, Baziotis $\mathrm{N}$. The role of $99 \mathrm{mTc}(\mathrm{V})$-DMSA scan as compared to $99 \mathrm{mTc}-\mathrm{MDP}$ and $\mathrm{CT}$ scans in imaging the primary tumor and metastases of osteosarcoma. Hell.J.Nucl.Med. $2005 ; 8(3): 162-4$.

7. Athanasoulis T, Koutsikos J, Moulopoulos LA, Tsiouris S, Dimopoulos MA, Zerva C. Reverse of the differential uptake intensity of Tc-99m MIBI and Tc-99m V-DMSA by multiple myeloma lesions in response to therapy. Clin. Nucl.Med. 2003;28(8):631-5.

8. Athanasoulis T, Koutsikos J, Zerva C, Horiuchi Suzuki K, Saji H, Ohta H. What is the source of the skeletal affinity of $99 \mathrm{mTc}-\mathrm{V}-\mathrm{DMSA}$ ? Eur. J. Nucl. Med. Mol. Imaging 2004;31(12):1673-6.

9. Durie BGM, Kyle RA, Belch A, Bensinger W, Blade J, Boccadoro $\mathrm{M}$, et al. Myeloma management guidelines: A consensus report from the Scientific Advisors of the International Myeloma Foundation. Hematol.J. 2003;4(6):379-98

10. (10) Alper E, Gurel M, Evrensel T, Ozkocaman V, Akbunar T, Demiray M. 99mTc-MIBI scintigraphy in untreated stage III multiple myeloma: Comparison with $\mathrm{X}$-ray skeletal survey and bone scintigraphy. Nucl.Med. Commun. 2003;24(5):537-42.

11. Ohta H, Endo K, Fujita T, Konishi J, Torizuka K, Horiuchi $\mathrm{K}$, et al. Clinical evaluation of tumour imaging using 99 $\mathrm{Tc}(\mathrm{V}) \mathrm{m}$ dimercaptosuccinic acid, a new tumour-seeking agent. Nucl.Med.Commun. 1988;9(2):105-16.

12. Pace L, Catalano L, Pinto A, De Renzo A, Di Gennaro F, Califano C, et al. Different patterns of technetium-99m sestamibi uptake in multiple myeloma. Eur.J.Nucl.Med. 1998;25(7):714-20.
13. Horiuchi Suzuki K, Konno A, Ueda M, Fukuda Y, Nishio $\mathrm{S}$, Hashimoto $\mathrm{K}$, et al. Skeletal affinity of Tc(V)-DMS is bone cell mediated and $\mathrm{pH}$ dependent. Eur.J.Nucl.Med. Mol.Imaging 2004;31(3):388-98.

14. Dimopoulos M, Terpos E, Comenzo RL, Tosi P, Beksac $\mathrm{M}$, Sezer $\mathrm{O}$, et al. International myeloma working group consensus statement and guidelines regarding the current role of imaging techniques in the diagnosis and monitoring of multiple myeloma. Leukemia 2009;23(9):1545-56.

15. Horiuchi K, Saji H, Yokoyama A. Tc(V)-DMS tumor localization mechanism: A pH-sensitive Tc(V)-DMSenhanced target/nontarget radio by glucose-mediated acidosis. Nucl.Med.Biol. 1998;25(6):549-55.

16. Denoyer D, Perek N, Le Jeune N, Frere D, Dubois F. Evidence that $99 \mathrm{mTc}-(\mathrm{V})$-DMSA uptake is mediated by $\mathrm{NaPi}$ cotransporter type III in tumour cell lines. Eur.J.Nucl. Med.Mol.Imaging 2004;31(1):77-84.

17. Stroobants S, Goeminne J, Seegers M, Dimitrijevic S, Dupont P, Nuyts J, et al. 18FDG-Positron emission tomography for the early prediction of response in advanced soft tissue sarcoma treated with imatinib mesylate (Glivec ${ }^{\circledR}$ ). Eur.J.Cancer 2003;39(14):2012-20.

18. Koutsikos J, Athanasoulis T, Anagnostopoulos A, Velidaki A, Passadi M, Dimopoulos MA, et al. Combined use of 99mTc-sestamibi and 99mTc-V-DMSA in the assessment of chemotherapy effectiveness in patients with multiple myeloma. J.Nucl.Med. 2005;46(6):978-82.

19. Piwnica Worms D. Characterization of multidrug resistance P-glycoprotein transport function with an organotechnetium cation. Biochemistry 1995;34(38):12210-20.

20. Perek N, Koumanov F, Denoyer D, Boudard D, Dubois F. Modulation of the multidrug resistance of glioma by glutathione levels depletion - Interaction with TC-99Msestamibi and TC-99M-tetrofosmin. Cancer Biother. Radiopharm. 2002;17(3):291-302.

21. Denoyer D, Perek N, Le Jeune N, Frère D, Dubois F. The Multidrug Resistance of In Vitro Tumor Cell Lines Derived from Human Breast Carcinoma MCF-7 Does Not Influence Pentavalent Technetium-99m-Dimercaptosuccinic Acid Uptake. Cancer Biother. Radiopharma. 2003; 18(5):791-801.

22. Mele A, Offidani M, Visani G, Marconi M, Cambioli F, Nonni M, et al. Technetium-99m sestamibi scintigraphy is sensitive and specific for the staging and the follow-up of patients with multiple myeloma: A multicentre study on 397 scans. Br.J.Haematol. 2007;136(5):729-35.

23. Svaldi M, Tappa C, Gebert U, Bettini D, Fabris P, Franzelin $\mathrm{F}$, et al. Technetium-99m-sestamibi scintigraphy: An alternative approach for diagnosis and follow-up of active myeloma lesions after high-dose chemotherapy and autologous stem cell transplantation. Ann.Hematol. 2001;80(7):393-7.

24. Balleari E, Villa $G$, Garrè $S$, Ghirlanda $P$, Agnese $G$, Carletto M, et al. Technetium-99m-sestaMIBI scintigraphy in multiple myeloma and related gammopathies: A useful tool for the identification and follow-up of myeloma bone disease. Haematologica 2001;86(1):78-84

25. Fonti R, Salvatore B, Quarantelli M, Sirignano C, Segreto $\mathrm{S}$, Petruzziello F, et al. 18F-FDG PET/CT, 99mTc-MIBI and MRI in evaluation of patients with multiple myeloma. J.Nucl.Med. 2008;49(2):195-200. 